\title{
Distance to Thrombus in Endovascular Treatment of Middle Cerebral Artery M1 Occlusion Predicts Recanalization Success and Clinical Outcome
}

\author{
Nihat Sengeze, $\mathrm{MD}^{1 *}$; Semih Giray, $\mathrm{MD}^{2}$ \\ ${ }^{1}$ Department of Neurology, Suleyman Demirel University Hospital, Isparta, Turkey \\ ${ }^{2}$ Department of Neurology, Gaziantep University Hospital, Gaziantep, Turkey
}

\begin{abstract}
Background: The occlusion site of the cerebral artery can help to determine recanalization success, treatment and prognosis in acute stroke patients. In current studies, different measurement techniques and different length values have been considered. We aimed to determine the relationship between the location of occlusion and recanalization success following endovascular therapy of acute middle cerebral artery (MCA) M1 occlusion.

Methods: This study was conducted from January 2015 to March 2019. The "M1 distance-to-thrombus length" was determined on curve-linear reformat reconstruction of the MCA, and measured from the center of internal carotid artery (ICA) bifurcation to the beginning of the thrombus on digital subtraction angiography (DSA). A successful recanalization was defined as $\geq$ modified thrombolysis in cerebral infarction $(\mathrm{mTICl}) 2 \mathrm{~b}$ and full recanalization as $\mathrm{mTICl} 3$. Evaluation of patients at the end of the third month was carried out with modified Rankin Scale (mRS) and mortality.

Results: We eventually included 95 patients treated with endovascular therapy. The patients with distance to thrombus (DT) $\leq 13.2 \mathrm{~mm}$ showed significantly higher rates of full recanalization (AUC $=0.639 \pm 0.06 ; P=0.014,95 \%$ confidence interval $[\mathrm{CI}]$ ). Additionally, DT could predict successful recanalization with an AUC of 0.639 . The possibility to distinguish unsuccessful recanalization cases after the endovascular treatment by considering DT had $85.7 \%$ sensitivity $(95 \% \mathrm{Cl})$. Of the $82(86.3 \%)$ patients who were treated with successful recanalization ( $\geq \mathrm{mTICI} 2 \mathrm{~b}), 46(48.4 \%)$ achieved $\mathrm{mRS}(0-3)$ and $38(40 \%)$ expired at the end of the 3 months.

Conclusion: Shorter DT was associated with higher rate of full recanalization (mTICl 3) after endovascular therapy. Having a longer DT reduces the chance of successful recanalization without distal embolism. However, there was no statistically significant effect for DT on a favorable outcome at third months or mortality with endovascular treatment of MCA M1 occlusions.

Keywords: Endovascular therapy, Middle cerebral artery, Thrombectomy, Thrombosis

Cite this article as: Sengeze N, Giray S. Distance to Thrombus in endovascular treatment of middle cerebral artery M1 occlusion predicts recanalization success and clinical outcome. Arch Iran Med. 2021;24(2):113-117. doi: 10.34172/aim.2021.17.
\end{abstract}

Received: January 5, 2020, Accepted: June 29, 2020, ePublished: February 1, 2021

\section{Introduction}

The middle cerebral artery (MCA) thrombus distance from internal carotid artery (ICA) bifurcation may be a quantifiable method for assessing the location of occlusive thrombus. Recent studies in acute ischemic stroke patients treated with intravenous (IV) thrombolysis (IVT) have shown that a shorter distance-to-thrombus (DT) is associated with higher rates of death and disability and larger diffusion-perfusion mismatch volumes on concurrent magnetic resonance imaging (MRI). ${ }^{1,2}$

Mechanical thrombectomy has been of clinical value in selected patients with acute stroke secondary to large vessel occlusion based on recent endovascular trials. Despite this treatment, close to half of the patients did not have acceptable clinical outcomes. ${ }^{3}$

Several landmark randomized clinical trials established the clinical efficacy of mechanical revascularization compared with TV tissue-type plasminogen activator. ${ }^{4-6}$
Zaidat et al showed that achievement of complete revascularization with a single thrombectomy device pass is associated with significantly higher rates of good clinical outcome. ${ }^{7}$ Therefore, recanalization as early as possible and on the first attempt the main goal of the endovascular treatment of stroke.

In this study, we aimed to investigate the correlation between the distance to thrombus (DT) measurement with digital subtraction angiography (DSA), recanalization success and clinical outcomes in endovascular therapy of MCA M1 occlusion.

\section{Materials and Methods}

Patient and Public Involvement

This study was a retrospective analysis of data in the stroke registry of Gaziantep University Hospital from January 2015 to March 2019. All stroke patients admitted to the hospital emergency department who had MCA M1 
occlusion and were admitted for endovascular treatment were included in this study, no patient was excluded from the study. Baseline and angiographic parameters were extracted from a prospectively collected database. Inclusion criteria were based on embolectomy using thrombectomy devices, in patients aged $\geq 18$ years with M1 occlusions and proper angiographic documentation of the procedure. In this procedure, distance to thrombus from ICA bifurcation to MCA M1 was detected after post-thrombectomy recanalization view of DSA.

In our study, we used the modified Rankin Scale (mRS) to evaluate the clinical outcome at the end of the third month after stroke. The mRS assesses disability in patients who have suffered from stroke and is compared over time to check for recovery and degree of continued disability. A score of 0 indicates no disability, 5 shows disability requiring constant care for all needs; and 6 denotes death.

Patients with tandem occlusions of ICA and MCA, double occlusion of MCA and anterior cerebral artery, and those with repeat stroke/reocclusion within 3 months of the presentation were excluded from the analysis. The National Institutes of Health Stroke Scale (NIHSS) score on admission and 3-month follow-up mRS were retrieved from the stroke registry and electronic medical records. A favorable outcome was defined by both third month mRS scores of (0-2) and (0-3).

\section{Treatment and Image Analysis}

The protocol for endovascular treatment of ischemic stroke patients during the timespan of this study has been described as IV thrombolytic therapy $(0.9 \mathrm{mg} / \mathrm{kg})$ if the patients were eligible. Furthermore, additional endovascular treatment [intra-arterial recombinant tissue plasminogen activator (rt-PA) and/or thrombectomy] was performed on those patients who had received IV thrombolysis, but had severe clinical symptoms (NIHSS score $\geq 10$ ) and large artery occlusion on admission. Recanalization success was evaluated based on the modified thrombolysis in cerebral infarction (mTICI) scoring system. Reperfusion results after endovascular therapy are usually reported using the mTICI score. The score was developed from the original Thrombolysis in
Cerebral Infarction scale by a consensus group in 2013 . Independent assessors at a DSA laboratory determined the mTICI score by reviewing the angiograms at the end of endovascular treatment. Successful recanalization was defined with scores of $\mathrm{mTICI} \geq 2 \mathrm{~b}$ and full recanalization with mTICI 3.

The "M1 distance-to-thrombus length" was determined on curve-linear reformat reconstruction of the MCA, and measured from the center of ICA bifurcation to the beginning of the thrombus on the anterior-posterior view of DSA (Figure 1A-1C).

In our study, the DT length was measured as a continuous variable using the DSA. Then, the operating point from the receiver operating characteristic (ROC) analysis was applied for dichotomization of the study cohort to emphasize on the difference between post endovascular treatment recanalization success rates of patient with proximal versus distal M1 occlusions.

\section{Statistical Methods}

The normality of distribution of continuous variables was tested using the Shaphiro Wilk test. The Mann Whitney U test was used to compare the two groups for non-normal numerical variables. The chi-square test was applied to investigate the relationship between categorical variables. A ROC curve analysis was performed, and the "cutoff point" was determined for dichotomization of the M1 DT length. In order to determine the best cutoff point, it was taken as the cutoff point for ROC analysis where the sensitivity + specificity value was the greatest. Frequency, percentage (\%) and median (interquartile range) were given as descriptive statistics. Statistical analysis was performed using SPSS for Windows version 23.0 and a $P$ value $<0.05$ was considered as statistically significant.

\section{Results}

We eventually included 95 patients (mean age $62.8 \pm$ 13.6; 62.1\% [59/95] female; mean NIHSS $16.4 \pm 3.6$ ) treated with endovascular therapy in MCA M1 occlusion. Forty-seven of the 95 patients (49.5\%) had right-sided hemiparesis due to stroke. In total, 82 patients $(86.3 \%)$ were treated with successful recanalization ( $\geq$ TICI $2 \mathrm{~b}$ ) at
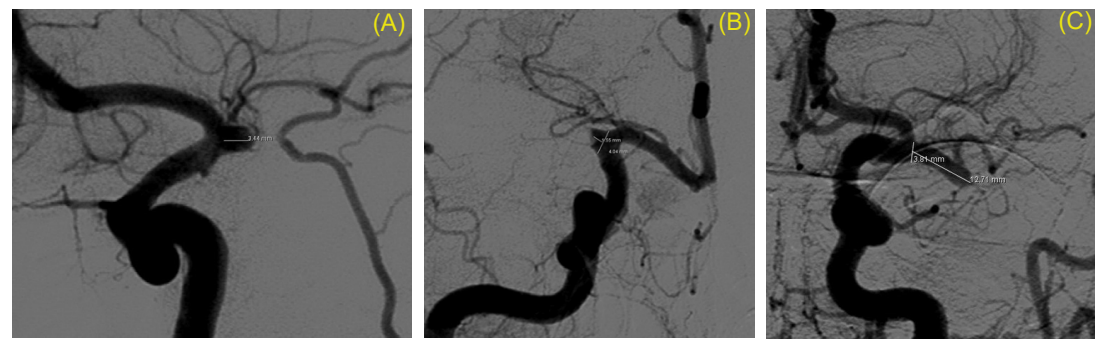

Figure 1. (A, B) The Short Distance to Thrombus on DSA Anterior-Posterior View. (C) An Example of Long Distance from Carotid T to Thrombus on DSA AnteriorPosterior View. 
the end of the endovascular therapy. In total, 28 patients $(29.5 \%)$ achieved a mRS of (0-2) at 3 months, 46 patients (48.4\%) achieved an mRS of (0-3) at 3 months (for favorable outcome) and 38 patients (40\%) expired at the end of the 3 months.

Regarding recanalization outcomes, patients with DT $\leq 13.2 \mathrm{~mm}$ showed significantly higher rates of full recanalization mTICI 3 (AUC $=0.639 \pm 0.06 ; P=$ $0.014)$. There is a possibility to distinguish unsuccessful recanalization cases after endovascular treatment by considering the DT $(85.7 \%$ sensitivity; $95 \% \mathrm{CI}=69.7-$ 95.2) (Figure 2).

The optimal cutoff point of distance to thrombus to predict recanalization achievement was determined according to the Youden's J index. In our sample, DT had sensitivity and specificity for predicting successful recanalization $(85.7 \%$ and $45.0 \%$, respectively).

The demographic data of both study groups are presented in Table 1 and comparison of demographic and clinical characteristics of patients by distance of thrombus from ICA bifurcation to M1 is presented in Table 2 .

\section{Discussion}

We found that the chance of full recanalization (mTICI 3) was higher among patients with $\mathrm{M} 1$ occlusion of $\leq 13.2$ $\mathrm{mm}$ from ICA bifurcation compared with those with DT of $>13.2 \mathrm{~mm}$ on admission DSA $(P=0.014)$. However, there was no statistically significant relationship between DT and successful recanalization ( $\geq$ mTICI $2 b$ ). In other words, having a high DT reduces the chance of successful recanalization without distal embolism. However, there was no significant correlation between DT and rate of favorable outcome. Anatomical studies have shown that

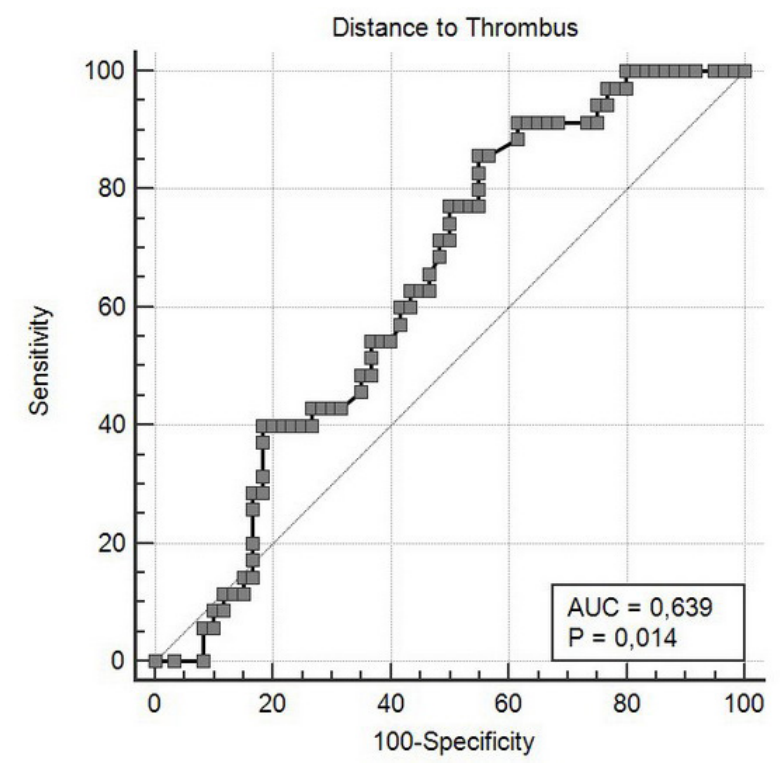

Figure 2. Discriminative Ability Presented as Receiver Operating Curve of Distance to Thrombus to Predict Recanalization Success at the End of the Endovascular Treatment.

the length of the MCA M1 segment varies between 11-20 $\mathrm{mm}$ and lenticulostriate vessels arise between $3-11 \mathrm{~mm} .^{8}$ As shown in some clinical studies, the distance from the carotid $\mathrm{T}$ to the beginning of the thrombus measured in millimeters along the MCA on a CT angiogram or DSA should be guiding for recanalization success in large artery occlusions. ${ }^{1,9}$ Lobsien et al noted that a combination of mechanical thrombectomy and IVT had more successful clinical outcomes than only IVT in patients with MCA M1 proximal occlusion (DT<16 mm). They defined DT as a new term to represent the occlusion location in

Table 1. Demographic Data of the Study Groups

\begin{tabular}{|c|c|c|c|c|}
\hline \multirow[t]{2}{*}{ Variables } & \multicolumn{4}{|c|}{ DT } \\
\hline & $\leq 13.2 \mathrm{~mm}(\mathrm{n}=63)$ & $>13.2 \mathrm{~mm}(\mathrm{n}=32)$ & Total $(n=95)$ & $P$ Value \\
\hline Age & $62(50-73)$ & $65(58-73)$ & $63(56-73)$ & 0.410 \\
\hline NIHSS & $18(14-19)$ & $16(13-18)$ & $16(14-19)$ & 0.057 \\
\hline Serum glucose & $157(118-187)$ & $134(112-165)$ & $146(116-178)$ & 0.184 \\
\hline Hemoglobin & $12.6(11.2-4.3)$ & $12.9(10.7-14)$ & $12.7(11.1-14.3)$ & 0.900 \\
\hline RDW & $14.4(13.7-15.3)$ & $14(13.2-14.6)$ & $14.2(13.6-15)$ & 0.065 \\
\hline Leukocyte & 9990 (8010-13800) & $10280(7130-11805)$ & $10050(7630-12810)$ & 0.526 \\
\hline BUN & $17.9(14.0-23.8)$ & $18.6(15.3-33.0)$ & $18.4(14.8-26.1)$ & 0.454 \\
\hline Creatinine & $0.78(0.63-1.0)$ & $0.80(0.59-0.91)$ & $0.80(0.6-1.0)$ & 0.591 \\
\hline Platelet & $258(206-310)$ & 237 (199-295) & $251(200-309)$ & 0.361 \\
\hline Sex, female & $42(66.7)$ & $17(53.1)$ & $59(62.1)$ & 0.198 \\
\hline Stroke side, right & $28(44.4)$ & $19(59.4)$ & $47(49.5)$ & 0.169 \\
\hline Antiplatelet drug use & $10(15.9)$ & $8(25)$ & $18(18.9)$ & 0.283 \\
\hline Atrial fibrillation & $22(34.9)$ & $10(31.3)$ & $32(33.7)$ & 0.721 \\
\hline Hypertension & $46(73)$ & $21(65.5)$ & $28(29.5)$ & 0.455 \\
\hline Cigarette smoking & $13(20.6)$ & $8(25)$ & $21(22.1)$ & 0.628 \\
\hline Diabetes mellitus & $22(34.9)$ & $6(18.8)$ & $28(29.5)$ & 0.102 \\
\hline History of coronary bypass & $3(4.8)$ & $1(3.1)$ & $4(4.2)$ & 0.707 \\
\hline Coroner artery disease & $13(20.6)$ & 7 (21.9) & $20(21.1)$ & 0.889 \\
\hline History of stroke & $4(6.3)$ & $3(9.4)$ & $7(7.4)$ & 0.594 \\
\hline
\end{tabular}

NIHSS, National Institutes of Health Stroke Scale; BUN, blood urine nitrogen; RDW, red distribution width; DT, distance-to-thrombus. 
Table 2. Comparison of Clinical Characteristics of Patients by Distance of Thrombus

\begin{tabular}{|c|c|c|c|c|}
\hline \multirow{3}{*}{ Clinical Characteristics } & \multicolumn{4}{|c|}{ DT } \\
\hline & $\leq 13.2 \mathrm{~mm}(\mathrm{n}=63)$ & $>13.2 \mathrm{~mm}(\mathrm{n}=32)$ & \multirow{2}{*}{$P$ Value } & \multirow{2}{*}{ Prevalence Difference $(\%)(95 \% \mathrm{Cl})$} \\
\hline & No. $(\%)$ & No. $(\%)$ & & \\
\hline IV thrombolytic treatment & $12(19)$ & $8(25)$ & 0.501 & $6(1.3-13.3)$ \\
\hline IA thrombolytic treatment & $45(71.4)$ & $26(81.3)$ & 0.298 & $9.9(6.1-13.78)$ \\
\hline Mechanical thrombectomy & $58(92.1)$ & $31(96.9)$ & 0.362 & $4.8(1.3-8.2)$ \\
\hline Successful recanalization (mTICI $\geq 2 b)$ & $53(84.1)$ & $29(90.6)$ & 0.384 & $6.5(2.8-10.1)$ \\
\hline Full recanalization $(\mathrm{mTICl} 3)$ & $30(47.6)$ & $5(15.6)$ & 0.014 & $32(26.4-37.5)$ \\
\hline $\mathrm{mRS}(0-2)$ & $16(25.4)$ & $12(37.5)$ & 0.221 & $12.1(5.9-18.2)$ \\
\hline mRS (0-3) & $27(42.9)$ & $19(59.4)$ & 0.128 & $16.5(11.6-21.3)$ \\
\hline Mortality in 3 months & $29(46)$ & $9(28.1)$ & 0.092 & $17.9(12.5-23.2)$ \\
\hline
\end{tabular}

IA, intra-arterial; IV, intravenous; mRS, modified Rankin Scale; $\mathrm{mTICl}$, modified thrombolysis in cerebral Infarction; Cl, confidence interval; DT, distance-tothrombus.

their studies. They claimed that there have been very few studies which used this evaluation and clinical results of IVT in MCA M1 occlusions were largely dependent on the location of occlusion. ${ }^{9}$

Clot length has been shown to affect the success of recanalization. Kamalian et al reported that thin-section non-contrast computed tomography images can be used to measure hyperdense clot length in acute ischemic stroke. They claimed that clot length ( $\geq 8$ - $\mathrm{mm}$ clot) measurement may be critical for triaging proximal MCA occlusions. By removing $25 \%$ to $30 \%$ of M1 clots that are short and likely to respond to IVT alone, patients who may benefit from catheter-based therapy may be rapidly triaged to the interventional suite. ${ }^{10}$ In our study, considering this issue from a different perspective, we also aimed to show that the distance of the clot from vascular branching is an effective factor for recanalization.

In long occlusions, the results of recanalization by IVT are also poor; proximal occlusions of the MCA are associated with worse patient outcomes. Consequently, a new analysis of the precise thrombus localization in acute MCA occlusions, the distance to thrombus, was established by Friedrich et al. They claimed that it was an easy-to-apply measurement of the length from the carotid $\mathrm{T}$ to the occlusion site in MCA stroke on computed tomography angiography. Gawlitza et al also attempted to validate the prognostic value of the DT on magnetic resonance angiography (MRA) in another study. They claimed that for a DT $<22 \mathrm{~mm}$, the likelihood of an unfavorable outcome (90d mRS 3-6 or NIHSS score improvement of $\leq 10$ points on discharge) was $>50 \%$. Initial diffusion tensor imaging lesion volumes showed no correlation with the outcome. DT on MRA was reliably measurable, correlated inversely with FLAIR-vascular hyperintensities, and predicted outcome in patients with acute MCA occlusion treated with IVT.,11

In our study, DT $(\leq 13.16 \mathrm{~mm})$ had a higher sensitivity $(85.7 \%)$ for predicting full recanalization (mTICI 3) which differed from this study. Besides, the DT length in our study was similar to what was mentioned in other studies. ${ }^{1,9}$ Pavabvash et al also investigated the thrombus localization and recanalization success in 34 MCA M1 occlusion patients on admission CT angiography. They indicated that the rate of successful recanalization was higher among patients with M1 DT of $\leq 6 \mathrm{~mm}$ with an odds ratio of $8(P=0.023)$. However, a M1 DT of $\leq 6 \mathrm{~mm}$ was not associated with a higher rate of favorable outcome. They achieved successful angiographic recanalization $(\geq$ mTICI $2 b)$ in $24(71 \%)$ out of 34 patients. ${ }^{12}$ In summary, existing studies have shown that when the studies are examined in terms of DT, there are quite variable results in terms of distance. Some of the reasons for this discrepancy include differences in the design of these studies, techniques used for measurement (DSA, CT angiography or MRA) and patient selection. As it is known, among all radiological methods, endovascular evaluation with DSA is the best measurement method. Here, we reported that measurement using endovascular evaluation predicts a different value than other studies.

Freiderich et al analyzed the DT parameter with preinterventional CT or MRI in 212 MCA M1 occlusions for predicting basal ganglia infarction of lenticulostriate arteries. They showed that the exact location of the arterial occlusion measured by DT in MCA M1 stroke can predict the lenticulostriate area and striatocapsular region infarction with high sensitivity and specificity $(P<0.001) .{ }^{13}$ This suggests that DT and its association with the salvage of lenticulostriate arteries area are also important entities in the endovascular treatment strategy. Thus, these inform the decision regarding how aggressive treatment will be the choice in patients with more distal occlusion.

In conclusion, DT is seen as an important parameter in endovascular treatment of stroke patients. As known, it is important in endovascular therapy of stroke that the stent-retriever (SR) must extend from the upper division to the proximal part of the clot to cover the long clots for M1 occlusions. So, the choice of SR length should also be according to these criteria. DT is also one of the criteria to be considered in this regard. Therefore, the neuro-interventionalist should be prepared with 
multiple endovascular techniques, SR or catheter selection and others for successful recanalization and less distal embolism.

The endovascular treatment of acute stroke is a very hot and popular topic. Our study population can be considered sufficient to yield strong conclusions. The results of the current study have clinical benefits regarding which cases may be challenging in terms of recanalization, and guiding the technique and procedure to be applied in cases. The other strengths of the study are that we addressed only a specific case study group, not all stroke cases (only MCA occlusion); all cases were processed in the same DSA laboratory by the same two neuro-interventionalists, and the distance to thrombus was measured by two independent neuro-interventionalists.

Our research had limitations; the study had a retrospective design. There was no clot histology data to further characterize the relationship about clot type; thus, a study about histological properties of clots may be interesting as regards their effect on recanalization.

In conclusion, shorter distance to thrombus of MCA M1 occlusion was associated with higher rate of full recanalization (mTICI 3) after endovascular therapy. Longer distance of M1 thrombus from the ICA bifurcation was associated with a poor rate of recanalization. Having a high DT reduces the chance of successful recanalization without distal embolism. However, there was no statistically significant effect for DT on favorable outcomes (mRS) at third months or mortality with endovascular treatment of MCA M1 occlusions.

\section{Authors' Contribution}

All authors had substantial contributions to the conception or design of the work and also to the acquisition, analysis, or interpretation of the data as well as drafting and revising the paper.

\section{Conflict of Interest Disclosures}

The authors declare that there is not any conflict of interests.

\section{Ethical Statement}

The study was approved by the Medicine Faculty Ethics Committee of Gaziantep University (2019/161).

\section{References}

1. Friedrich B, Gawlitza M, Schob S, Hobohm C, Raviolo M, Hoffmann KT, et al. Distance to thrombus in acute middle cerebral artery occlusion: a predictor of outcome after intravenous thrombolysis for acute ischemic stroke. Stroke.
2015;46(3):692-6. doi: 10.1161/STROKEAHA.114.008454.

2. Gawlitza M, Friedrich B, Hobohm C, Schaudinn A, Schob S, Quäschling $U$, et al. Distance to Thrombus in Acute Middle Cerebral Artery Occlusion Predicts Target Mismatch and Ischemic Penumbra. J Stroke Cerebrovasc Dis. 2016;25(2):298305. doi: 10.1016/j.jstrokecerebrovasdis.2015.09.032.

3. Chamorro Á, Blasco J, López A, Amaro S, Román LS, Llull L, et al. Complete reperfusion is required for maximal benefits of mechanical thrombectomy in stroke patients. Sci Rep. 2017;14;7(1):11636. doi: 10.1038/s41598-017-11946-y.

4. Berkhemer OA, Fransen PS, Beumer D, van den Berg LA, Lingsma HF, Yoo AJ, et al MR CLEAN Investigators. A randomized trial of intraarterial treatment for acute ischemic stroke. N Engl J Med. 2015;1;372(1):11-20. doi: 10.1056/ NEJMoa1411587.

5. Goyal M, Demchuk AM, Menon BK, Eesa M, Rempel JL, Thornton J, et al ESCAPE Trial Investigators. Randomized assessment of rapid endovascular treatment of ischemic stroke. N Engl J Med. 2015;12;372(11):1019-30. doi: 10.1056/ NEJMoa1414905.

6. Jovin TG, Chamorro A, Cobo E, de Miquel MA, Molina CA, Rovira A, et al REVASCAT Trial Investigators. Thrombectomy within 8 hours after symptom onset in ischemic stroke. N Engl J Med. 2015;11;372(24):2296-306. doi: 10.1056/ NEJMoa1503780.

7. Zaidat OO, Castonguay AC, Linfante I, Gupta R, Martin CO, Holloway WE, et al. First Pass Effect: A New Measure for Stroke Thrombectomy Devices. Stroke. 2018;49(3):660-6. doi: 10.1161/STROKEAHA.117.020315.

8. Pai SB, Varma RG, Kulkarni RN. Microsurgical anatomy of the middle cerebral artery. Neurol India. 2005;53(2):186-90. doi: 10.4103/0028-3886.16406

9. Lobsien D, Gawlitza M, Schaudinn A, Schob S, Hobohm C, Fritzsch D, et al. Mechanical thrombectomy versus systemic thrombolysis in MCA stroke: a distance to thrombus-based outcome analysis. J Neurointerv Surg. 2016;8(9):878-82. doi: 10.1136/neurintsurg-2015-011964.

10. Kamalian S, Morais LT, Pomerantz SR, Aceves M, Sit SP, Bose $A$, et al. Clot length distribution and predictors in anterior circulation stroke: implications for intra-arterial therapy. Stroke. 2013;44(12):3553-6. doi: 10.1161/STROKEAHA.113.003079.

11. Gawlitza M, Friedrich B, Quäschling U, Schob S, Schaudinn A, Hobohm C, et al. Distance to thrombus on MR angiography predicts outcome of middle cerebral artery occlusion treated with IV thrombolysis. Neuroradiology. 2015;57(10):991-7. doi: 10.1007/s00234-015-1558-9.

12. Pavabvash S, Taleb S, Majidi S, Qureshi Al. Correlation of Acute M1 Middle Cerebral Artery Thrombus Location with Endovascular Treatment Success and Clinical Outcome. J Vasc Interv Neurol. 2017;9(3):17-22.

13. Friedrich B, Lobsien D, Maegerlein C, Wunderlich S, Zimmer C, Kaesmacher J, et al. Distance to Thrombus in acute middle cerebral artery stroke predicts basal ganglia infarction after mechanical thrombectomy. Oncotarget. 2016;27;7(52):85813-8. doi: 10.18632/oncotarget.13280. 